\section{Posição socioeconômica no curso de vida e comportamentos de risco relacionados à saúde: ELSA-Brasil}

\section{Lifetime socioeconomic status and health-related risk behaviors: the ELSA-Brazil study}
Posición socioeconómica en el curso de vida y comportamientos de riesgo relacionados con la salud: ELSA-Brasil

Jéssica Costa Faleiro 1

Luana Giatti 1

Sandhi Maria Barreto 1

Lidyane do Valle Camelo 1

Rosane Härter Griep 2

Joanna M. N. Guimarães 3

Maria de Jesus Mendes da Fonseca ${ }^{3}$

Dóra Chor 3

Maria da Conceição Almeida Chagas 4

doi: 10.1590/0102-311X00017916

\section{Resumo}

Nosso objetivo foi investigar a associação da posição socioeconômica no curso de vida e da mobilidade social intrageracional com o baixo consumo de frutas e hortaliças, inatividade física no lazer e tabagismo entre 13.216 homens e mulheres participantes da linha de base do ELSA-Brasil (2008-2010). A posição socioeconômica na infância, juventude e vida adulta foi aferida pela escolaridade materna, classe sócio-ocupacional da primeira ocupação e classe sócio-ocupacional da ocupação atual, respectivamente. Desvantagens sociais na vida adulta foram consistentemente associadas à maior prevalência dos três comportamentos analisados em homens e mulheres. Entretanto, a posição socioeconômica na juventude e infância foi associada aos comportamentos de forma menos consistente. Por exemplo, enquanto a baixa escolaridade materna reduziu a chance de tabagismo passado (mulheres) e atual (homens e mulheres), ela foi associada à maior chance de inatividade física no lazer entre as mulheres. Já a exposição à baixa posição socioeconômica na juventude aumentou as chances de tabagismo passado (homens e mulheres) e atual (mulheres). A análise das trajetórias sociais deu suporte adicional à maior importância das desvantagens na vida adulta para comportamentos de risco, já que apenas indivíduos que ascenderam para a classe socio-ocupacional alta não apresentaram maior chance destes comportamentos quando comparados aos participantes que sempre pertenceram à classe sócio-ocupacional alta. Nossos resultados apontam que desvantagens socioeconômicas na vida adulta parecem ser mais relevantes para comportamentos de risco do que as desvantagens na infância e adolescência.

Classe Social; Mobilidade Social; Comportamentos Saudáveis

\section{Correspondência}

L. Giatti

Faculdade de Medicina, Universidade Federal de Minas Gerais. Av. Alfredo Balena 190, Belo Horizonte, MG 30350-150, Brasil. luana.giatti@gmail.com

1 Universidade Federal de Minas Gerais, Belo Horizonte, Brasil. 2 Instituto Oswaldo Cruz, Fundação Oswaldo Cruz, Rio de Janeiro, Brasil.

3 Escola Nacional de Saúde Pública Sergio Arouca, Fundação Oswaldo Cruz, Rio de Janeiro, Brasil.

4 Instituto Gonçalo Moniz, Fundação Oswaldo Cruz, Salvador, Brasil. 


\section{Introdução}

Comportamentos não saudáveis são mais comumente observados em indivíduos de baixa posição socioeconômica 1,2 , e esta tem sido um dos mecanismos que explica a relação entre a exposição à adversidade socioeconômica e maior morbimortalidade por doenças crônicas não transmissíveis (DCNT) 3,4. Os comportamentos de risco relacionados à saúde, como o baixo consumo de frutas e hortaliças, inatividade física e tabagismo, são os principais fatores de risco modificáveis para morbidade e mortalidade por DCNT 5 . A redução da prevalência desses comportamentos de risco é essencial para diminuir a carga de morbimortalidade por essas doenças.

A posição socioeconômica que o indivíduo ocupa influencia o modo de vida e o trabalho, e pode criar vulnerabilidades ao desenvolvimento de doenças 6 . Sabe-se que as pessoas não estão distribuídas aleatoriamente em circunstâncias saudáveis e não saudáveis 7 , e que a capacidade e a possibilidade de escolhas saudáveis são fortemente influenciadas pela posição socioeconômica em cada etapa da vida 8,9 . Estudos prévios já investigaram a relação entre a posição econômica na vida adulta e comportamentos relacionados à saúde, e mostraram que a exposição às adversidades sociais está associada à maior prevalência destes comportamentos 10,11. É possível, entretanto, que os comportamentos relacionados à saúde também sejam influenciados pela experiência de adversidades socioeconômicas em períodos anteriores do curso de vida, como a infância e a juventude. Estudos sugerem que a exposição a adversidades socioeconômicas na juventude 12 e, sobretudo na infância 13,14,15,16, podem impactar negativamente no padrão da dieta 13,14 , prática de atividade física 15 e consumo de tabaco 12,16, independentemente da posição socioeconômica atingida na vida adulta. Entretanto, diferentemente das DCNT que não são reversíveis, comportamentos podem ser alterados. Portanto, é possível que mudanças na posição socioeconômica ao longo da vida possam induzir modificações em comportamentos de risco. Por exemplo, indivíduos que migram de posição socioeconômica mais baixa na infância para uma mais alta na vida adulta podem adotar comportamentos mais frequentes no grupo social atual 15,17. Entretanto, poucos trabalhos investigaram se, e em que direção, mudanças na trajetória ocupacional, ou seja, a mobilidade social estaria associada à prevalência de comportamentos de risco 18 .

As associações entre a posição socioeconômica no curso da vida e comportamentos também podem variar segundo gênero ${ }^{19}$. Estudos sugerem que a posição socioeconômica na infância tende a ser mais importante na determinação dos comportamentos em mulheres, já a posição socioeconômica na vida adulta seria mais relevante para os homens 14,16, mas as evidências quanto a diferenças por gênero nestas associações ainda são escassas e inconsistentes. Por exemplo, a associação das desvantagens socioeconômicas no início da vida com o tabagismo atual, ex-tabagismo, obesidade 16 e pior padrão de consumo alimentar 14 mantiveram-se significantes apenas em mulheres, após ajuste pela condição socioeconômica atual. No entanto, diversos trabalhos mostram associações entre desvantagens na posição socioeconômica em etapas anteriores e o baixo consumo de frutas e vegetais $13 \mathrm{e}$ ex-tabagismo 20 em homens, independentemente da posição socioeconômica na vida adulta. Além do mais, não sabemos se há diferenças de gênero na relação entre mobilidade na PSE e comportamentos de risco.

O objetivo do presente trabalho foi investigar se a pior posição socio-econômica em cada uma das etapas do curso de vida (infância, juventude e vida adulta) está associada com o baixo consumo de frutas e hortaliças, inatividade física no lazer e tabagismo, independentemente da posição socieconômica nas demais etapas em homens e mulheres participantes da linha de base do Estudo Longitudinal de Saúde do Adulto (ELSA-Brasil). Adicionalmente, verificamos se a mobilidade intrageracional na classe social estava associada a esses comportamentos.

\section{Método}

Trata-se de uma análise empregando abordagem de curso de vida utilizando dados da linha de base do ELSA-Brasil, realizada entre 2008-2010. O ELSA-Brasil é uma coorte multicêntrica de 15.105 servidores públicos voluntários, com idades entre 35 e 74 anos, ativos ou aposentados de instituições de ensino superior e pesquisa de seis capitais de estados brasileiros: Belo Horizonte (Minas Gerais), 
Porto Alegre (Rio Grande do Sul), Rio de Janeiro, Salvador (Bahia), São Paulo e Vitória (Espírito Santo). Todos os participantes realizaram entrevistas face a face por meio de questionário organizado em módulos temáticos, testado em estudos piloto e realizado por entrevistadores treinados e certificados 21 . O estudo foi aprovado pelos comitês de ética em pesquisa de cada uma das instituições envolvidas e todos os participantes assinaram Termo de Consentimento Livre e Esclarecido.

Dos 15.105 servidores públicos participantes do ELSA-Brasil, foram excluídos da presente análise aqueles com diagnóstico médico referido de diabetes $(\mathrm{n}=1.322)$ e doenças cardiovasculares (infarto agudo do miocárdio, acidente vascular encefálico, insuficiência cardíaca ou revascularização do miocárdio) ( $\mathrm{n}=567)$, uma vez que estas condições podem induzir mudanças nos comportamentos relacionados à saúde por orientações médicas 22. Assim, 1.889 participantes foram excluídos e a amostra analítica foi composta por 13.216 pessoas.

\section{Variáveis do estudo}

\section{- Variáveis respostas}

1) Baixo consumo de frutas e hortaliças (verduras e legumes) obtido por meio das perguntas: Com que frequência o(a) Sr(a). costuma comer verduras e legumes crus, cozidos ou refogados, sem incluir batatas, mandioca/aipim, inhame e cará?, e Com que frequência o(a) Sr(a). costuma comer frutas, sem incluir sucos de frutas? As opções de resposta foram: "mais de 3x/dia", "2-3x/dia", "1x/dia", "5-6x/semana", "2-4x/semana", "1x/ semana", "1-3x/mês", "nunca/quase nunca". Os participantes foram categorizados de forma binária, sendo considerado baixo consumo de frutas e hortaliças o consumo inferior a uma vez ao dia.

2) Inatividade física no lazer foi mensurada por meio do tempo de atividade física no lazer da versão longa do Questionário Internacional de Atividade Física (IPAQ) 23, que avalia a frequência semanal e duração da prática de atividade física leve (caminhada), moderada (nadar ou pedalar em ritmo médio, praticar esportes por diversão etc.) e vigorosa (correr, fazer ginástica em academia, pedalar em ritmo rápido, praticar esportes competitivos etc.). Essa variável foi categorizada de forma binária, sendo considerados inativos fisicamente aqueles que praticavam menos que $150 \mathrm{~min}$ de atividade física moderada ou menos que $75 \mathrm{~min}$ de atividade física vigorosa por semana, segundo recomendações da Organização Mundial da Saúde (OMS) 24.

3) Tabagismo mensurado por meio de duas perguntas: $O(a)$ senhor(a) é ou já foi fumante, ou seja, já fumou pelo menos 100 cigarros (cinco maços de cigarros) ao longo da sua vida?, e $O($ a) senhor(a) fuma cigarros atualmente? Os participantes foram categorizados em: tabagista (aqueles que declararam ter fumado pelo menos 100 cigarros ao longo da vida e que fumavam no momento da realização da pesquisa); ex-tabagista (os que afirmaram ter fumado pelo menos 100 cigarros ao longo da vida e que não fumavam no momento da realização da pesquisa); e os que nunca fumaram (aqueles que não fumaram pelo menos cinco maços ou 100 cigarros ao longo da vida).

\section{- Indicadores de posição socioeconômica no curso de vida}

A posição socioeconômica na infância foi aferida pela escolaridade materna, categorizada em "nunca estudou", "1-7 anos", "8-10 anos" e "maior ou igual a 11 anos", e mensurada retrospectivamente com base na pergunta: Qual é o grau de instrução de sua mãe?

A posição socioedonômica na juventude foi aferida pela classe sócio-ocupacional da primeira ocupação do participante. Essa classe é uma medida sumária baseada em três aspectos: a ocupação, o rendimento esperado com base no nível de educação (valor médio de mercado) e a renda observada. Em primeiro lugar, o status socioeconômico foi estimado usando-se a média entre os rendimentos esperado e o observado para cada participante. Em segundo, para cada título ocupacional, a média do escore do status socioeconômico dos indivíduos foi estimada em obter o status socioeconômico ocupacional. Em seguida, utilizando-se esses escores de status socioeconômico ocupacional, foram definidos os estratos sócio-ocupacionais agrupando os escores de maneira a obter uma mínima variância intraestrato e o máximo de variação entre os estratos. Esses escores foram classificados em sete níveis, e para a análise atual foram resumidos em cinco categorias: alta (alta superior e alta inferior), média superior (média superior e média média), média inferior, baixa superior e baixa inferior. 
A posição socioeconômica na vida adulta foi aferida pela classe sócio-ocupacional atual do participante e obtida usando-se a mesma abordagem utilizada para obter a classe sócio-ocupacional da primeira ocupação.

A mobilidade social intrageracional foi representada pela mudança entre a classe socio-ocupacional da primeira ocupação e a classe sócio-ocupacional da ocupação atual. Para facilitar a análise da mobilidade, as variáveis classe sócio-ocupacional na juventude e classe socio-ocupacional atual foram categorizadas em: alta (alta superior e alta inferior), média (média superior, média média e média inferior) e baixa (baixa superior e baixa inferior). Dessa forma, a variável mobilidade foi classificada em seis níveis: alta - > alta (alta classe social na juventude e alta classe social atual), baixa/média - > alta (baixa ou média classe social na juventude e alta classe social atual), média - > média (média classe social na juventude e média classe social atual), baixa $->$ média (baixa classe social na juventude e média classe social atual), alta/média - > baixa (alta e média classe social na juventude e baixa classe social atual), alta - > média (alta classe social na juventude e média classe social atual), baixa - > baixa (baixa classe social na juventude e baixa classe social atual). Devido ao pequeno número de indivíduos na categoria alta - > média $(H=30 ; M=65)$, esta categoria foi excluída da análise.

\section{- Covariáveis}

As seguintes variáveis foram utilizadas como covariáveis: sexo, idade em faixas etárias (35-44, 45-54, 55-64, 65-74 anos) e raça/cor autodeclarada (branca, parda, preta, amarela e indígena).

\section{Análise dos dados}

Todas as análises foram conduzidas separadamente para homens e mulheres, já que estudos prévios apontam diferenças de gênero nas associações investigadas 16,19. Foi realizada a descrição da população do trabalho por meio de proporções. A análise univariável entre os indicadores de posição socioeconômica e comportamentos de risco relacionados à saúde foi realizada por meio do teste de qui-quadrado de Pearson, com nível de 5\% de significância. O nível de significância de $20 \%$ foi utilizado para a seleção das variáveis que foram inseridas na análise múltipla. Modelos de regressão logística binomial (baixo consumo de frutas e hortaliças, inatividade física no lazer) e modelos de regressão logística multinomial (tabagismo) foram usados nas análises múltiplas para a obtenção do odds ratio ajustado com intervalo de 95\% de confiança (IC95\%). Foi estimada a força de associação entre cada indicador de posição socioeconômica (escolaridade materna, classe sócio-ocupacional na juventude, classe sócio-ocupacional na vida adulta) e cada comportamento de saúde separadamente. Foram realizados ajustes sequenciais da seguinte forma: estimativa bruta (Modelo 0) (modelos não apresentados), inclusão de idade e raça/cor (Modelo 1) e ao final, inclusão dos dois outros indicadores de posição (Modelo 2). Para verificar a força da associação entre a mobilidade intrageracional na classe sócio-ocupacional e cada comportamento separadamente foi realizado apenas o ajuste por idade e raça/cor (Modelo 1). As variáveis retidas nos modelos finais foram as que permaneceram associadas às variáveis respostas em um nível de significância de $5 \%$. A análise foi realizada utilizando-se o programa Stata 12.0 (StataCorp LP, College Station, Estados Unidos).

\section{Resultados}

Dos 13.216 participantes da linha de base do ELSA-Brasil incluídos neste trabalho, mais de 50\% tinham mãe com menos de oito anos de escolaridade, das quais cerca de $12 \%$ nunca frequentaram a escola. A maioria dos participantes pertencia à classe sócio-ocupacional baixa na juventude. Por outro lado, cerca de um terço dos participantes foi classificado como pertencentes à classe sócio-ocupacional alta na vida adulta. Consequentemente, verificou-se que cerca de $50 \%$ dos participantes apresentaram mobilidade social intrageracional ascendente. Os homens registraram maior prevalência de baixo consumo de frutas e hortaliças $(67,7 \%$ versus $51,7 \%)$ e o mesmo padrão foi encontrado para tabagismo atual (14,4\% versus $12,2 \%)$; já as mulheres apresentaram maior prevalência de inatividade física no lazer (78,3\% versus 70,8\%) (Tabela 1$)$. 


\section{Tabela 1}

Descrição das características da população do estudo. Estudo Longitudinal de Saúde do Adulto (ELSA-Brasil), 2008-2010.

\begin{tabular}{|c|c|c|}
\hline & $\begin{array}{c}\text { Masculino }(n=5.864) \\
n(\%)\end{array}$ & $\begin{array}{c}\text { Feminino }(n=7.352) \\
n(\%)\end{array}$ \\
\hline \multicolumn{3}{|c|}{$\begin{array}{l}\text { Características sociodemográficas } \\
\text { Idade (anos) }\end{array}$} \\
\hline $35-44$ & $1.500(25,6)$ & $1.726(23,5)$ \\
\hline $45-54$ & $2.388(40,7)$ & $3.005(40,9)$ \\
\hline $55-64$ & $1.438(24,5)$ & $2.023(27,5)$ \\
\hline $65-74$ & $538(9,17)$ & $598(8,13)$ \\
\hline \multicolumn{3}{|l|}{ Raça/Cor } \\
\hline Branco & $3.113(53,8)$ & $3.853(52,9)$ \\
\hline Pardo & $1.738(30,0)$ & $1.934(26,6)$ \\
\hline Preto & $763(1,7)$ & $1.225(16,8)$ \\
\hline Amarelo & $99(1,7)$ & $213(2,9)$ \\
\hline Indígena & $75(1,3)$ & $54(0,7)$ \\
\hline \multicolumn{3}{|l|}{ Posição socioeconômica } \\
\hline \multicolumn{3}{|c|}{ Escolaridade materna (anos de estudo) } \\
\hline$\geq 11$ & $1.495(26,2)$ & $1.704(23,6)$ \\
\hline $8-10$ & $1.099(19,3)$ & $1.433(19,8)$ \\
\hline $1-7$ & $2.328(40,8)$ & $3.191(44,2)$ \\
\hline 0 & $778(13,6)$ & $898(12,4)$ \\
\hline \multicolumn{3}{|c|}{ Classe sócio-ocupacional na primeira ocupação } \\
\hline Alta & $320(6,2)$ & $457(7,6)$ \\
\hline Média superior & $745(14,5)$ & $1.107(18,4)$ \\
\hline Média inferior & $718(14,0)$ & $1.214(20,2)$ \\
\hline Baixa superior & $2.423(47,1)$ & $2.483(41,4)$ \\
\hline Baixa inferior & $936(18,2)$ & $739(12,3)$ \\
\hline \multicolumn{3}{|c|}{ Classe sócio-ocupacional atual } \\
\hline Alta & $2.135(37,0)$ & $2.319(32,1)$ \\
\hline Média superior & $1.272(22,0)$ & $1.901(26,3)$ \\
\hline Média inferior & $745(12,9)$ & $1.585(21,9)$ \\
\hline Baixa superior & $342(5,9)$ & $802(11,1)$ \\
\hline Baixa inferior & $1.278(21,1)$ & $622(8,6)$ \\
\hline \multicolumn{3}{|c|}{ Mobilidade social intrageracional } \\
\hline Alta - > Alta & $287(5,7)$ & $374(6,4)$ \\
\hline Baixa/média - > Alta & $1.403(27,9)$ & $1.404(24,0)$ \\
\hline Média - > Média & $507(10,1)$ & $1.138(19,5)$ \\
\hline Baixa - > Média & $1.292(25,7)$ & $1.692(29,0)$ \\
\hline Alta/média - > Baixa & $197(3,9)$ & $263(4,5)$ \\
\hline Baixa - > Baixa & $1.349(26,8)$ & $968(16,6)$ \\
\hline \multicolumn{3}{|c|}{ Comportamentos de risco relacionados à saúde } \\
\hline \multicolumn{3}{|c|}{ Baixo consumo de frutas e hortaliças } \\
\hline Não & $1.892(32,3)$ & $3.541(48,3)$ \\
\hline Sim & $3.961(67,7)$ & $3.794(51,7)$ \\
\hline \multicolumn{3}{|l|}{ Inatividade física } \\
\hline Não & $1.687(29,2)$ & $1.568(21,7)$ \\
\hline Sim & $4.091(70,8)$ & $5.672(78,3)$ \\
\hline \multicolumn{3}{|l|}{ Tabagismo } \\
\hline Nunca fumou & $3.087(52,6)$ & $4.627(62,9)$ \\
\hline Ex-tabagista & $1.932(32,9)$ & $1.827(24,8)$ \\
\hline Tabagista & $845(14,4)$ & $898(12,2)$ \\
\hline
\end{tabular}

Nota: as diferenças nos totais ocorrem devido à perda de informação. 
Observou-se que quanto mais baixa a escolaridade da mãe, maiores foram as prevalências de baixo consumo de frutas e hortaliças e inatividade física no lazer em ambos os sexos, e de tabagismo atual em mulheres e ex-tabagismo entre os homens. Desvantagens socioeconômicas na juventude e na vida adulta também foram associadas a maiores prevalências de baixo consumo de frutas e hortaliças, inatividade física no lazer, tabagismo atual em homens e mulheres e ex-tabagismo em homens. No geral, observou-se que participantes de ambos os sexos com estabilidade na classe social baixa e com mobilidade descendente (da classe alta/média para a baixa) apresentaram maiores prevalências de comportamentos não saudáveis, já os que sempre pertenceram à classe social alta e aqueles com mobilidade ascendente (da classe baixa/média para a alta) apresentaram menores prevalências destes comportamentos (Tabela 2).

Em homens, após ajuste por idade e raça/cor, as desvantagens socioeconômicas na infância mantiveram-se associadas a maiores chances de baixo consumo de frutas e hortaliças, inatividade física no lazer e ex-tabagista (Modelo 1). Entretanto, após introduzir no modelo a posição socioeconômica na juventude e na fase adulta (Modelo 2), a menor escolaridade materna permaneceu estatisticamente associada apenas à menor chance de ser tabagista atual. Da mesma forma, a adversidade na juventude (classes baixa inferior e baixa superior), após ajuste pela posição socioeconômica na infância e vida adulta, permaneceu associada apenas à maior chance de ser ex-tabagista. Já a classe sócio-ocupacional atual, após todos os ajustes, manteve-se associada inversamente a maiores chances de baixo consumo de frutas e verduras, inatividade física no lazer, tabagismo atual e ex-tabagismo (Tabela 3).

Em mulheres, as desvantagens socioeconômicas na infância permaneceram associadas a maiores chances de baixo consumo de frutas e hortaliças e inatividade física no lazer após considerar a idade e a raça/cor (Modelo 1), mas após o ajuste simultâneo pela posição socioeconômica na juventude e vida adulta (Modelo 2), a associação foi significante apenas para inatividade física no lazer. Ao mesmo tempo, a baixa escolaridade materna foi associada menores chances de tabagismo atual e de ex-tabagismo. A classe sócio-ocupacional baixa na juventude apresentou associação com comportamentos de risco após ajuste por idade e raça/cor, esteve associada apenas à maior chance de tabagismo e extabagismo após considerar a posição socioeconômica na infância e juventude. Finalmente, a classe sócio-ocupacional atual foi a única que permaneceu inversamente associada a todos os comportamentos analisados no modelo final, com indicação de maior chance de baixo consumo de frutas e verduras, inatividade física no lazer e tabagismo atual, quanto mais baixa a classe social (Tabela 4).

Com relação à trajetória na classe sócio-ocupacional da juventude para a vida adulta, observamos que comparados aos que permaneceram estáveis na classe sócio-ocupacional alta, a estabilidade na classe sócio-ocupacional baixa, assim como os que descenderam para esta classe, apresentaram maiores chances de comportamentos de risco após ajustes (Modelo 1) em homens e mulheres. Adicionalmente, maiores chances de comportamentos de risco também foram observadas entre os participantes estáveis na classe sócio-ocupacional média e os que ascenderam para esta classe em ambos os sexos (Tabela 5).

\section{Discussão}

Nossos resultados apontam que a posição socioeconômica atual parece ter um papel mais relevante para a prevalência de comportamentos de risco para a saúde do que a posição socioeconômica na juventude e na infância, uma vez que desvantagens na vida adulta foram associadas à maior prevalência de todos os comportamentos analisados, independentemente da posição socioeconômica em fases anteriores da vida. Por outro lado, as circunstâncias socioeconômicas adversas na juventude e infância foram relacionadas de forma contraditória a alguns comportamentos. Enquanto a baixa escolaridade materna reduziu a chance de tabagismo passado (mulheres) e atual (homens e mulheres), ela foi associada à maior chance de inatividade física no lazer entre as mulheres. Já a exposição à baixa posição socioeconômica na juventude aumentou as chances de tabagismo passado (homens e mulheres) e atual (mulheres). As análises das trajetórias sociais dão suporte adicional à maior importância das desvantagens na vida adulta para a presença desses comportamentos de risco, uma vez que apenas indivíduos que ascenderam da classe baixa ou media para a classe alta não apresentaram chance diferente de 


\section{Tabela 2}

Prevalência de comportamentos relacionados à saúde de acordo com a posição socioeconômica estratificada por sexo. Estudo Longitudinal de Saúde do Adulto (ELSA-Brasil), 2008-2010.

\begin{tabular}{|c|c|c|c|c|c|c|c|c|}
\hline & \multirow{2}{*}{\multicolumn{2}{|c|}{$\begin{array}{l}\text { Baixo consumo de } \\
\text { frutas e hortaliças * }\end{array}$}} & \multirow{2}{*}{\multicolumn{2}{|c|}{ Inatividade física ** }} & \multicolumn{4}{|c|}{ Tagismo } \\
\hline & & & & & \multicolumn{2}{|c|}{ Ex-tabagista *** } & \multicolumn{2}{|c|}{ Tagismo \# } \\
\hline & Homens & Mulheres & Homens & Mulheres & Homens & Mulheres & Homens & Mulheres \\
\hline \multicolumn{9}{|c|}{ Escolaridade materna (anos de estudo) } \\
\hline$\geq 11$ & 63,0 & 46,6 & 65,3 & 69,0 & 29,6 & 27,5 & 13,2 & 12,4 \\
\hline $8-10$ & 65,7 & 51,7 & 68,5 & 80,0 & 30,8 & 25,0 & 13,4 & 11,2 \\
\hline $1-7$ & 69,1 & 52,9 & 71,9 & 80,1 & 32,9 & 23,0 & 15,1 & 11,9 \\
\hline 0 & 73,2 & 56,7 & 78,7 & 86,6 & 40,4 & 26,4 & 14,8 & 13,4 \\
\hline Valor de $\mathrm{p}$ & $<0,001$ & $<0,001$ & $<0,001$ & $<0,001$ & $<0,001$ & 0,214 & $<0,001$ & 0,214 \\
\hline \multicolumn{9}{|c|}{ Classe sócio-ocupacional na primeira } \\
\hline \multicolumn{9}{|l|}{ ocupação } \\
\hline Alta & 58,3 & 45,7 & 62,7 & 69,3 & 27,2 & 23,4 & 13,4 & 8,3 \\
\hline Média superior & 64,4 & 46,2 & 67,0 & 72,1 & 28,0 & 25,5 & 9,9 & 10,6 \\
\hline Média inferior & 66,5 & 51,4 & 67,8 & 79,5 & 32,0 & 24,3 & 12,4 & 11,8 \\
\hline Baixa superior & 70,6 & 54,2 & 71,9 & 82,2 & 35,3 & 26,0 & 17,0 & 13,3 \\
\hline Baixa inferior & 72,0 & 56,8 & 78,6 & 85,7 & 38,2 & 26,2 & 15,9 & 15,7 \\
\hline Valor de $\mathrm{p}$ & $<0,001$ & $<0,001$ & $<0,001$ & $<0,001$ & $<0,001$ & $<0,001$ & $<0,001$ & $<0,001$ \\
\hline \multicolumn{9}{|c|}{ Classe sócio-ocupacional atual } \\
\hline Alta & 56,0 & 42,7 & 64,0 & 69,4 & 31,4 & 25,4 & 8,7 & 8,6 \\
\hline Média superior & 73,9 & 55,9 & 69,1 & 77,5 & 29,1 & 24,7 & 14,6 & 13,1 \\
\hline Média inferior & 73,6 & 56,3 & 75,1 & 83,9 & 33,7 & 25 & 16,8 & 12,0 \\
\hline Baixa superior & 71,0 & 53,6 & 79,2 & 84,8 & 41,8 & 23,8 & 16,1 & 15,1 \\
\hline Baixa inferior & 77,1 & 60,32 & 79,4 & 93,3 & 35,9 & 23,9 & 22,1 & 20,1 \\
\hline Valor de p & $<0,001$ & $<0,001$ & $<0,001$ & $<0,001$ & $<0,001$ & $<0,001$ & $<0,001$ & $<0,001$ \\
\hline \multicolumn{9}{|c|}{ Mobilidade social intrageracional } \\
\hline Alta - > Alta & 57,7 & 44,9 & 61,2 & 68,7 & 27,5 & 22,2 & 11,5 & 8,0 \\
\hline Baixa/Média - > Alta & 56,8 & 41,8 & 64,2 & 71,0 & 33,4 & 26,4 & 9,0 & 9,1 \\
\hline Média - > Média & 74,1 & 54,9 & 70,6 & 79,0 & 27,4 & 24,8 & 11,8 & 11,9 \\
\hline Baixa - > Média & 73,6 & 56,9 & 72,1 & 82,1 & 33,4 & 25,9 & 16,4 & 12,8 \\
\hline Alta/Média - > Baixa & 78,7 & 48,3 & 75,0 & 84,7 & 31,5 & 22,0 & 18,8 & 14,8 \\
\hline Baixa - > Baixa & 75,4 & 58,3 & 79,6 & 90,6 & 38,3 & 25,4 & 20,8 & 18,5 \\
\hline Valor de $\mathrm{p}$ & $<0,001$ & $<0,001$ & $<0,001$ & $<0,001$ & $<0,001$ & $<0,001$ & $<0,001$ & $<0,001$ \\
\hline
\end{tabular}

* Baixo consumo de frutas e hortaliças: consumo inferior a uma vez ao dia;

** Inatividade física: < 150 minutos/semana de atividade física moderada ou < 75 minutos/semana de atividade física forte;

*** Ex-tabagista: consumo de pelo menos 100 cigarros ao longo da vida e não fuma atualmente;

\# Tabagista: consumo de pelo menos 100 cigarros ao longo da vida e fuma atualmente.

comportamentos de risco, quando comparados aos participantes que sempre pertenceram à classe sócio-ocupacional alta. A persistência, assim como a mobilidade para a classe sócio-ocupacional baixa, bem como a estabilidade e a mobilidade ascendente para a classe média, foram associadas à maior chance de comportamentos de risco.

Os diferenciais na ocorrência dos comportamentos estudados por gênero nesta coorte são consistentes com outros trabalhos, já que as mulheres neste estudo também tenderam a ser mais saudáveis do que os homens 10,11. Observou-se uma maior prevalência de comportamentos de risco relacionados à saúde entre os homens, exceto a inatividade física no lazer, que foi mais frequente dentre as mulheres 25. Dados da Pesquisa Nacional de Saúde (PNS-2013) também corroboram os diferenciais de gênero observados entre os participantes desta coorte 26. 
Tabela 3

Odds ratio e intervalo de 95\% de confiança da associação entre indicadores de posição socioeconômica e comportamentos relacionados à saúde em homens. Estudo Longitudinal de Saúde do Adulto (ELSA-Brasil), 2008-2010.

\begin{tabular}{|c|c|c|c|c|c|c|c|c|}
\hline & \multirow{2}{*}{\multicolumn{2}{|c|}{$\begin{array}{l}\text { Baixo consumo de frutas } \\
\text { e hortaliças }\end{array}$}} & \multirow{2}{*}{\multicolumn{2}{|c|}{ Inatividade física }} & \multicolumn{4}{|c|}{ Tabagismo } \\
\hline & & & & & \multicolumn{2}{|c|}{ Ex-tabagista } & \multicolumn{2}{|c|}{ Tabagismo } \\
\hline & Modelo 1 & Modelo 2 & Modelo 1 & Modelo 2 & Modelo 1 & Modelo 2 & Modelo 1 & Modelo 2 \\
\hline \multicolumn{9}{|c|}{$\begin{array}{l}\text { Escolaridade materna } \\
\text { (anos de estudo) }\end{array}$} \\
\hline$\geq 11$ & 1,00 & 1,00 & 1,00 & 1,00 & 1,00 & 1,00 & 1,00 & 1,00 \\
\hline $8-10$ & $\begin{array}{c}1,10 \\
(0,93-1,30)\end{array}$ & $\begin{array}{c}0,93 \\
(0,78-1,10)\end{array}$ & $\begin{array}{c}1,13 \\
(0,95-1,34)\end{array}$ & $\begin{array}{c}0,98 \\
(0,83-1,17)\end{array}$ & $\begin{array}{c}1,02 \\
(0,85-1,22)\end{array}$ & $\begin{array}{c}0,83 \\
(0,67-1,02)\end{array}$ & $\begin{array}{c}0,96 \\
(0,75-1,23)\end{array}$ & $\begin{array}{c}0,68 \\
(0,51-0,90)\end{array}$ \\
\hline $1-7$ & $\begin{array}{c}1,24 \\
(1,08-1,43)\end{array}$ & $\begin{array}{c}0,99 \\
(0,85-1,15)\end{array}$ & $\begin{array}{c}1,30 \\
(1,12-1,50)\end{array}$ & $\begin{array}{c}1,06 \\
(0,91-1,24)\end{array}$ & $\begin{array}{c}1,17 \\
(1,00-1,37)\end{array}$ & $\begin{array}{c}0,85 \\
(0,70-1,02)\end{array}$ & $\begin{array}{c}1,13 \\
(0,92-1,38)\end{array}$ & $\begin{array}{c}0,71 \\
(0,55-0,90)\end{array}$ \\
\hline 0 & $\begin{array}{c}1,54 \\
(1,26-1,89)\end{array}$ & $\begin{array}{c}1,07 \\
(0,85-1,33)\end{array}$ & $\begin{array}{c}1,71 \\
(1,38-2,12)\end{array}$ & $\begin{array}{c}1,23 \\
(0,98-1,55)\end{array}$ & $\begin{array}{c}1,45 \\
(1,18-1,79)\end{array}$ & $\begin{array}{c}0,93 \\
(0,72-1,18)\end{array}$ & $\begin{array}{c}1,16 \\
(0,88-1,53)\end{array}$ & $\begin{array}{c}0,58 \\
(0,42-0,81)\end{array}$ \\
\hline \multicolumn{9}{|l|}{$\begin{array}{l}\text { Classe sócio- } \\
\text { ocupacional na } \\
\text { primeira ocupação }\end{array}$} \\
\hline Alta & 1,00 & 1,00 & 1,00 & 1,00 & 1,00 & 1,00 & 1,00 & 1,00 \\
\hline Média superior & $\begin{array}{c}1,24 \\
(0,94-1,64)\end{array}$ & $\begin{array}{c}1,09 \\
(0,82-1,44)\end{array}$ & $\begin{array}{c}1,20 \\
(0,91-1,59)\end{array}$ & $\begin{array}{c}1,09 \\
(0,82-1,44)\end{array}$ & $\begin{array}{c}1,05 \\
(0,77-1,43)\end{array}$ & $\begin{array}{c}0,93 \\
(0,67-1,28)\end{array}$ & $\begin{array}{c}0,71 \\
(0,46-1,07)\end{array}$ & $\begin{array}{c}0,57 \\
(0,37-0,88)\end{array}$ \\
\hline Média inferior & $\begin{array}{c}1,28 \\
(0,97-1,69)\end{array}$ & $\begin{array}{c}1,00 \\
(0,75-1,33)\end{array}$ & $\begin{array}{c}1,23 \\
(0,92-1,63)\end{array}$ & $\begin{array}{c}1,01 \\
(0,75-1,34)\end{array}$ & $\begin{array}{c}1,40 \\
(1,02-1,92)\end{array}$ & $\begin{array}{c}1,26 \\
(0,91-1,75)\end{array}$ & $\begin{array}{c}0,97 \\
(0,64-1,47)\end{array}$ & $\begin{array}{c}0,70 \\
(0,45-1,08)\end{array}$ \\
\hline Baixa superior & $\begin{array}{c}1,49 \\
(1,16-1,90)\end{array}$ & $\begin{array}{c}1,05 \\
(0,81-1,36)\end{array}$ & $\begin{array}{c}1,51 \\
(1,17-1,94)\end{array}$ & $\begin{array}{c}1,10 \\
(0,85-1,44)\end{array}$ & $\begin{array}{c}1,96 \\
(1,48-2,61)\end{array}$ & $\begin{array}{c}1,63 \\
(1,21-2,21)\end{array}$ & $\begin{array}{c}1,60 \\
(1,11-2,28)\end{array}$ & $\begin{array}{c}1,04 \\
(0,70-1,55)\end{array}$ \\
\hline Baixa inferior & $\begin{array}{c}1,60 \\
(1,21-2,11)\end{array}$ & $\begin{array}{c}1,01 \\
(0,75-1,36)\end{array}$ & $\begin{array}{c}2,01 \\
(1,51-2,67)\end{array}$ & $\begin{array}{c}1,31 \\
(0,97-1,78)\end{array}$ & $\begin{array}{c}1,89 \\
(1,39-2,57)\end{array}$ & $\begin{array}{c}1,40 \\
(1,00-1,96)\end{array}$ & $\begin{array}{c}1,42 \\
(0,96-2,11)\end{array}$ & $\begin{array}{c}0,78 \\
(0,50-1,22)\end{array}$ \\
\hline \multicolumn{9}{|l|}{$\begin{array}{l}\text { Classe sócio- } \\
\text { ocupacional atual }\end{array}$} \\
\hline Alta & 1,00 & 1,00 & 1,00 & 1,00 & 1,00 & 1,00 & 1,00 & 1,00 \\
\hline Média superior & $\begin{array}{c}1,86 \\
(1,58-2,18)\end{array}$ & $\begin{array}{c}1,86 \\
(1,58-2,18)\end{array}$ & $\begin{array}{c}1,38 \\
(1,18-1,61)\end{array}$ & $\begin{array}{c}1,38 \\
(1,18-1,61)\end{array}$ & $\begin{array}{c}1,39 \\
(1,17-1,65)\end{array}$ & $\begin{array}{c}1,20 \\
(0,98-1,46)\end{array}$ & $\begin{array}{c}2,02 \\
(1,59-2,56)\end{array}$ & $\begin{array}{c}1,97 \\
(1,50-2,58)\end{array}$ \\
\hline Média inferior & $\begin{array}{c}1,84 \\
(1,51-2,23)\end{array}$ & $\begin{array}{c}1,84 \\
(1,51-2,23)\end{array}$ & $\begin{array}{c}1,83 \\
(1,51-2,21)\end{array}$ & $\begin{array}{c}1,83 \\
(1,51-2,21)\end{array}$ & $\begin{array}{c}1,71 \\
(1,39-2,10)\end{array}$ & $\begin{array}{c}1,54 \\
(1,22-1,93)\end{array}$ & $\begin{array}{c}2,56 \\
(1,95-3,35)\end{array}$ & $\begin{array}{c}2,41 \\
(1,76-3,29)\end{array}$ \\
\hline Baixa superior & $\begin{array}{c}1,94 \\
(1,50-2,52)\end{array}$ & $\begin{array}{c}1,94 \\
(1,50-2,52)\end{array}$ & $\begin{array}{c}2,02 \\
(1,52-2,67)\end{array}$ & $\begin{array}{c}2,02 \\
(1,52-2,67)\end{array}$ & $\begin{array}{c}1,80 \\
(1,38-2,34)\end{array}$ & $\begin{array}{c}1,49 \\
(1,10-2,00)\end{array}$ & $\begin{array}{c}2,56 \\
(1,78-3,66)\end{array}$ & $\begin{array}{c}2,32 \\
(1,53-3,50)\end{array}$ \\
\hline Baixa inferior & $\begin{array}{c}2,17 \\
(1,84-2,57)\end{array}$ & $\begin{array}{c}2,17 \\
(1,84-2,57)\end{array}$ & $\begin{array}{c}2,25 \\
(1,91-2,66)\end{array}$ & $\begin{array}{c}2,25 \\
(1,91-2,66)\end{array}$ & $\begin{array}{c}2,07 \\
(1,74-2,47)\end{array}$ & $\begin{array}{c}1,74 \\
(1,41-2,16)\end{array}$ & $\begin{array}{c}3,8 \\
(3,02-4,78)\end{array}$ & $\begin{array}{c}3,80 \\
(2,85-5,06)\end{array}$ \\
\hline
\end{tabular}

Modelo 1: ajustada por idade e raça; Modelo 2: Escolaridade materna: modelo 1 + classe socio-ocupacional na juventude e classe socio-ocupacional atual; Classe sócio-ocupacional na juventude: modelo 1 + escolaridade materna e classe socio-ocupacional atual; Classe sócio-ocupacional atual: modelo $1+$ escolaridade materna e classe socio-ocupacional na juventude.

Verificou-se um gradiente socioeconômico, de modo que quanto mais baixa a posição socioeconômica atual, maior a chance de baixo consumo de frutas e hortaliças, inatividade física no lazer e tabagismo atual, em ambos os sexos. Essas associações foram pouco atenuadas após o ajuste pela posição socioeconômica na infância e na juventude, reforçando o papel central das circunstâncias socioeconômicas na vida adulta para os comportamentos relacionados à saúde. Estudos prévios já apontaram associação entre desvantagem na posição socioeconômica e menor prevalência de atividade física no lazer 25 e consumo de frutas e hortaliças 27 , inclusive muitas destas associações foram independentes da posição socioeconômica na infância 13,15. Da mesma forma, a associação entre baixa posição socioeconômica atual e tabagismo é observada na literatura internacional 15,16 e nacional 17,28. 
Odds ratio e intervalo de $95 \%$ de confiança da associação entre indicadores de posição socioeconômica e comportamentos relacionados à saúde em mulheres. Estudo Longitudinal de Saúde do Adulto (ELSA-Brasil), 2008-2010.

\begin{tabular}{|c|c|c|c|c|c|c|c|c|}
\hline & \multirow{2}{*}{\multicolumn{2}{|c|}{$\begin{array}{c}\text { Baixo consumo de frutas } \\
\text { e hortaliças }\end{array}$}} & \multirow{2}{*}{\multicolumn{2}{|c|}{ Inatividade física }} & \multicolumn{4}{|c|}{ Tabagismo } \\
\hline & & & & & \multicolumn{2}{|c|}{ Ex-tabagista } & \multicolumn{2}{|c|}{ Tabagista } \\
\hline & Modelo 1 & Modelo 2 & Modelo 1 & Modelo 2 & Modelo 1 & Modelo 2 & Modelo 1 & Modelo 2 \\
\hline \multicolumn{9}{|c|}{$\begin{array}{l}\text { Escolaridade materna } \\
\text { (anos de estudo) }\end{array}$} \\
\hline$\geq 11$ & 1,00 & 1,00 & 1,00 & 1,00 & 1,00 & 1,00 & 1,00 & 1,0 \\
\hline $8-10$ & $\begin{array}{c}1,20 \\
(1,04-1,39)\end{array}$ & $\begin{array}{c}1,10 \\
(0,94-1,27)\end{array}$ & $\begin{array}{c}1,71 \\
(1,44-2,02)\end{array}$ & $\begin{array}{c}1,52 \\
(1,28-1,81)\end{array}$ & $\begin{array}{c}0,83 \\
(0,70-0,99)\end{array}$ & $\begin{array}{c}0,76 \\
(0,63-0,93)\end{array}$ & $\begin{array}{c}0,82 \\
(0,65-1,03)\end{array}$ & $\begin{array}{c}0,71 \\
(0,55-0,93)\end{array}$ \\
\hline $1-7$ & $\begin{array}{c}1,21 \\
(1,07-1,37)\end{array}$ & $\begin{array}{c}1,07 \\
(0,94-1,22)\end{array}$ & $\begin{array}{c}1,67 \\
(1,45-1,92)\end{array}$ & $\begin{array}{c}1,37 \\
(1,18-1,59)\end{array}$ & $\begin{array}{c}0,76 \\
(0,66-0,88)\end{array}$ & $\begin{array}{c}0,65 \\
(0,54-0,77)\end{array}$ & $\begin{array}{c}0,85 \\
(0,70-1,03)\end{array}$ & $\begin{array}{c}0,62 \\
(0,49-0,78)\end{array}$ \\
\hline 0 & $\begin{array}{c}1,42 \\
(1,19-1,69)\end{array}$ & $\begin{array}{c}1,17 \\
(0,97-1,41)\end{array}$ & $\begin{array}{c}2,50 \\
(1,99-3,13)\end{array}$ & $\begin{array}{c}1,59 \\
(1,25-2,03)\end{array}$ & $\begin{array}{c}0,91 \\
(0,75-1,11)\end{array}$ & $\begin{array}{c}0,66 \\
(0,52-0,85)\end{array}$ & $\begin{array}{c}1,03 \\
(0,80-1,34)\end{array}$ & $\begin{array}{c}0,62 \\
(0,45-0,85)\end{array}$ \\
\hline \multicolumn{9}{|l|}{$\begin{array}{l}\text { Classe sócio- } \\
\text { ocupacional na } \\
\text { primeira ocupação }\end{array}$} \\
\hline Alta & 1,00 & 1,00 & 1,00 & 1,00 & 1,00 & 1,00 & 1,00 & 1,00 \\
\hline Média superior & $\begin{array}{c}1,08 \\
(0,86-1,35)\end{array}$ & $\begin{array}{c}0,94 \\
(0,74-1,18)\end{array}$ & $\begin{array}{c}1,09 \\
(0,86-1,39)\end{array}$ & $\begin{array}{c}0,95 \\
(0,74-1,23)\end{array}$ & $\begin{array}{c}1,17 \\
(0,90-1,53)\end{array}$ & $\begin{array}{c}1,15 \\
(0,88-1,52)\end{array}$ & $\begin{array}{c}1,51 \\
(1,01-2,26)\end{array}$ & $\begin{array}{c}1,35 \\
(0,89-2,05)\end{array}$ \\
\hline Média inferior & $\begin{array}{c}1,17 \\
(0,94-1,46)\end{array}$ & $\begin{array}{c}0,95 \\
(0,75-1,20)\end{array}$ & $\begin{array}{c}1,61 \\
(1,25-2,06)\end{array}$ & $\begin{array}{c}1,17 \\
(0,90-1,52)\end{array}$ & $\begin{array}{c}1,16 \\
(0,89-1,51)\end{array}$ & $\begin{array}{c}1,19 \\
(0,90-1,58)\end{array}$ & $\begin{array}{c}1,63 \\
(1,10-2,41)\end{array}$ & $\begin{array}{c}1,43 \\
(0,94-2,17)\end{array}$ \\
\hline Baixa superior & $\begin{array}{c}1,27 \\
(1,03-1,56)\end{array}$ & $\begin{array}{c}1,00 \\
(0,80-1,25)\end{array}$ & $\begin{array}{c}1,83 \\
(1,45-2,30)\end{array}$ & $\begin{array}{c}1,20 \\
(0,94-1,55)\end{array}$ & $\begin{array}{c}1,36 \\
(1,07-1,74)\end{array}$ & $\begin{array}{c}1,40 \\
(1,07-1,82)\end{array}$ & $\begin{array}{c}2,01 \\
(1,39-2,91)\end{array}$ & $\begin{array}{c}1,66 \\
(1,11-2,48)\end{array}$ \\
\hline Baixa inferior & $\begin{array}{c}1,43 \\
(1,12-1,83)\end{array}$ & $\begin{array}{c}1,05 \\
(0,80-1,37)\end{array}$ & $\begin{array}{c}2,30 \\
(1,71-3,10)\end{array}$ & $\begin{array}{c}1,04 \\
(0,74-1,46)\end{array}$ & $\begin{array}{c}1,39 \\
(1,04-1,86)\end{array}$ & $\begin{array}{c}1,43 \\
(1,03-1,99)\end{array}$ & $\begin{array}{c}2,42 \\
(1,60-3,66)\end{array}$ & $\begin{array}{c}1,72 \\
(1,08-2,75)\end{array}$ \\
\hline \multicolumn{9}{|l|}{$\begin{array}{l}\text { Classe sócio- } \\
\text { ocupacional atual }\end{array}$} \\
\hline Alta & 1,00 & 1,00 & 1,00 & 1,00 & 1,00 & 1,00 & 1,00 & 1,00 \\
\hline Média superior & $\begin{array}{c}1,47 \\
(1,29-1,67)\end{array}$ & $\begin{array}{c}1,47 \\
(1,29-1,67)\end{array}$ & $\begin{array}{c}1,46 \\
(1,27-1,68)\end{array}$ & $\begin{array}{c}1,38 \\
(1,19-1,60)\end{array}$ & $\begin{array}{c}1,16 \\
(0,99-1,34)\end{array}$ & $\begin{array}{c}1,17 \\
(0,98-1,39)\end{array}$ & $\begin{array}{c}1,68 \\
(1,36-2,07)\end{array}$ & $\begin{array}{c}1,56 \\
(1,22-2,00)\end{array}$ \\
\hline Média inferior & $\begin{array}{c}1,46 \\
(1,28-1,67)\end{array}$ & $\begin{array}{c}1,46 \\
(1,28-1,67)\end{array}$ & $\begin{array}{c}2,10 \\
(1,78-2,48)\end{array}$ & $\begin{array}{c}1,87 \\
(1,57-2,23)\end{array}$ & $\begin{array}{c}1,16 \\
(0,99-1,36)\end{array}$ & $\begin{array}{c}1,24 \\
(1,03-1,51)\end{array}$ & $\begin{array}{c}1,51 \\
(1,21-1,90)\end{array}$ & $\begin{array}{c}1,50 \\
(1,14-1,97)\end{array}$ \\
\hline Baixa superior & $\begin{array}{c}1,48 \\
(1,25-1,74)\end{array}$ & $\begin{array}{c}1,48 \\
(1,25-1,74)\end{array}$ & $\begin{array}{c}2,28 \\
(1,84-2,83)\end{array}$ & $\begin{array}{c}2,04 \\
(1,63-2,55)\end{array}$ & $\begin{array}{c}1,00 \\
(0,82-1,22)\end{array}$ & $\begin{array}{c}1,06 \\
(0,84-1,34)\end{array}$ & $\begin{array}{c}1,95 \\
(1,51-2,52)\end{array}$ & $\begin{array}{c}1,94 \\
(1,43-2,63)\end{array}$ \\
\hline Baixa inferior & $\begin{array}{c}1,69 \\
(1,40-2,04)\end{array}$ & $\begin{array}{c}1,69 \\
(1,40-2,04)\end{array}$ & $\begin{array}{c}5,54 \\
(3,95-7,77)\end{array}$ & $\begin{array}{c}4,57 \\
(3,23-6,49)\end{array}$ & $\begin{array}{c}1,24(0,99- \\
1,56)\end{array}$ & $\begin{array}{c}1,31 \\
(1,00-1,72)\end{array}$ & $\begin{array}{c}2,92 \\
(2,23-3,81)\end{array}$ & $\begin{array}{c}2,82 \\
(2,02-3,94)\end{array}$ \\
\hline
\end{tabular}

Modelo 1: ajustada por idade e raça; Modelo 2: Escolaridade materna: modelo 1 + classe socio-ocupacional na juventude e classe sócio-ocupacional atual; Classe sócio-ocupacional na juventude: modelo 1 + escolaridade materna e classe sócio-ocupacional atual; Classe sócio-ocupacional atual: modelo $1+$ escolaridade materna e classe socio-ocupacional na juventude.

Entretanto, homens e mulheres das classes sócio-ocupacionais baixa inferior e média inferior apresentaram maior chance de ex-tabagismo. Possivelmente, esses indivíduos tiveram maior chance de serem tabagistas no passado. Apesar das taxas de abandono do tabagismo serem menores em populações que vivem desvantagens socioeconômicas 29 , neste estudo não foi analisado o índice de cessação do tabagismo, e sim a chance de ser ex-tabagista comparada à de nunca fumar. Isso pode explicar a associação observada entre exposição à desvantagem na vida adulta e ex-tabagismo. Além disso, a associação entre piores condições socioeconômicas e maior chance de ser ex-tabagista já foi descrita em outros estudos 30, inclusive em análise prévia do ELSA-Brasil 31. 


\section{Tabela 5}

Odds ratio e intervalo de 95\% de confiança da associação entre mobilidade social intrageracional e comportamentos relacionados à saúde em ambos os sexos. Estudo Longitudinal de Saúde do Adulto (ELSA-Brasil), 2008-2010.

\begin{tabular}{|c|c|c|c|c|}
\hline & \multirow{2}{*}{$\begin{array}{c}\text { Baixo consumo de frutas e } \\
\text { hortaliças * }\end{array}$} & \multirow[t]{2}{*}{ Inatividade física ** } & \multicolumn{2}{|c|}{ Tabagismo } \\
\hline & & & Ex-tabagista *** & Tabagista \# \\
\hline \multicolumn{5}{|l|}{ Homens } \\
\hline Alta - > Alta & 1,00 & 1,00 & 1,00 & 1,00 \\
\hline Baixa/Média - > Alta & $1,00(0,77-1,30)$ & $1,13(0,87-1,48)$ & $1,27(0,94-1,72)$ & $0,83(0,54-1,26)$ \\
\hline Média - > Média & $1,83(1,33-2,51)$ & $1,67(1,22-2,28)$ & $1,42(1,00-2,02)$ & $1,20(0,74-1,92)$ \\
\hline Baixa - > Média & $1,75(1,33-2,31)$ & $1,75(1,34-2,30)$ & $2,00(1,46-2,73)$ & $1,91(1,26-2,90)$ \\
\hline Alta/Média - > Baixa & $2,32(1,52-3,54)$ & $1,92(1,28-2,89)$ & $1,69(1,10-2,61)$ & $2,00(1,16-3,46)$ \\
\hline Baixa - > Baixa & $1,99(1,51-2,63)$ & $2,51(1,91-3,31)$ & $2,53(1,86-3,46)$ & $2,84(1,88-4,28)$ \\
\hline \multicolumn{5}{|l|}{ Mulheres } \\
\hline Alta - > Alta & 1,00 & 1,00 & 1,00 & 1,00 \\
\hline Baixa/Média - > Alta & $0,92(0,73-1,17)$ & $1,06(0,82-1,37)$ & $1,28(0,96-1,69)$ & $1,37(0,88-2,12)$ \\
\hline Média - > Média & $1,38(1,08-1,75)$ & $1,60(1,22-2,09)$ & $1,31(0,98-1,76)$ & $1,81(1,17-2,79)$ \\
\hline Baixa - > Média & $1,44(1,14-1,82)$ & $1,86(1,44-2,41)$ & $1,49(1,13-1,97)$ & $2,08(1,36-3,17)$ \\
\hline Alta/Média - > Baixa & $1,12(0,81-1,55)$ & $2,33(1,55-3,51)$ & $1,10(0,74-1,63)$ & $2,28(1,34-3,86)$ \\
\hline Baixa - > Baixa & $1,62(1,26-2,08)$ & $3,90(2,84-5,35)$ & $1,48(1,10-2,00)$ & $3,27(2,12-5,05)$ \\
\hline
\end{tabular}

* Baixo consumo de frutas e hortaliças: consumo inferior a uma vez ao dia;

** Inatividade física: < 150 minutos/semana de atividade física moderada ou < 75 minutos/semana de atividade física forte;

*** Ex-tabagista: consumo de pelo menos 100 cigarros ao longo da vida e não fuma atualmente;

\# Tabagista: consumo de pelo menos 100 cigarros ao longo da vida e fuma atualmente.

Nota: ajustado por idade e raça.

Os comportamentos relacionados à saúde também são, em grande medida, influenciados pelos contextos material, físico e social em que os indivíduos vivem. Aqueles em desvantagens na posição socioeconômica moram em vizinhanças com menos recursos e oportunidades que estimulariam a adoção de comportamentos saudáveis, como menor disponibilidade de estabelecimentos para a compra de alimentos saudáveis 32 . A restrição no acesso, somada ao fato desses alimentos serem perecíveis e frequentemente caros 33 , pode contribuir para o consumo de alimentos industrializados em populações de baixa posição socioeconômica em detrimento de frutas e hortaliças 34 . O contexto desfavorável na vizinhança, como atributos estéticos 35 , assim como a presença de maiores índices de violência, pior iluminação e falta de locais públicos seguros para a realização de atividades físicas tendem a impactar negativamente na realização dessas atividades no lazer em áreas residenciais mais pobres 36 . Além disso, indivíduos em piores condições socioeconômicas geralmente não têm acesso à atividade física em clubes e academias 37 . O ambiente pode também ser mais ou menos permissível ao tabagismo; comunidades de baixa posição socioeconômica podem estar mais expostas à publicidade e venda do tabaco 38 .

Os comportamentos são também moldados pelo ambiente e redes de relações sociais, os grupos sociais podem compartilhar preferências em relação, por exemplo, aos hábitos alimentares 8. Considere-se ainda, que as classes sociais mais altas têm maior acesso a conhecimentos sobre os fatores de risco para a saúde e, em função dos maiores recursos objetivos e subjetivos, são as que mais se beneficiam de novas estratégias e práticas que promovem a saúde ${ }^{39}$. As pessoas em desvantagem socioeconômica, além de terem mais restrição à educação em saúde, tendem a aderir menos a programas de prevenção e promoção de comportamentos saudáveis 22 .

A relação entre posição socioeconômica na infância e comportamentos de saúde não se mostrou consistente. Em geral, as associações entre a posição socioeconômica na infância e comportamentos não foram independentes da posição socioeconômica na vida adulta. Outros estudos também observaram que o tabagismo e a inatividade física no lazer foram mais fortemente associados com a posição socioeconômica na vida adulta do que na infância 15,40,41. 
Um achado que chama atenção na presente análise foi a associação entre a baixa escolaridade materna e a menor chance de tabagismo atual e passado em mulheres, e atual em homens. Esses achados são discordantes de estudos longitudinais que identificaram maior tabagismo entre aqueles de classe social baixa na infância, após controlar pela escolaridade atual 20,41. Em parte, essa diferença pode ser atribuída às mudanças do tabagismo no contexto brasileiro. Estudos brasileiros mostram que o tabagismo foi mais prevalente entre os de posição socioeconômica mais alta até 1989, quando teve início o programa de controle do tabagismo 42 . Os participantes do presente trabalho nasceram entre 1934 e 1975, portanto, a prevalência de mães fumantes provavelmente era menor entre as de baixa renda. Como o contágio pela exposição familiar é um componente importante da propagação do tabagismo, e os participantes do ELSA-Brasil nasceram em uma época em que este hábito era mais prevalente entre mulheres de posição socioeconômica mais alta, acreditamos que isto pode explicar a menor chance de tabagismo atual e passado entre participantes com mães de menor escolaridade. Assim, é possível que a menor prevalência de tabagismo em mães de menor escolaridade tenha influenciado este comportamento protetor, uma vez que crescer em um ambiente livre de tabaco está associado à menor probabilidade de tabagismo na vida adulta 12,43.

Verificamos ainda que a desvantagem social na infância apresentou associação independente com inatividade física no lazer entre as mulheres, resultado já observado em estudo prévio ${ }^{15}$. Não podemos afirmar com esses achados, mas é possível que a escolaridade materna tenha maior relevância para os comportamentos de saúde entre as mulheres do que entre os homens. Por exemplo, Chapman et al. 19 observaram que parte da associação entre posição socioeconômica na infância e a ganho de peso na vida adulta foi parcialmente mediada pela posição socioeconômica na vida adulta em mulheres, e completamente mediada pela posição socioeconômica na vida adulta nos homens.

Grande parte da iniciação nos comportamentos de risco como o tabagismo ocorre na adolescência, fase da vida em que outras redes sociais passam a ter maior influência sobre atitudes e comportamentos 12. Poucos estudos, entretanto, investigaram se as circunstâncias socioeconômicas na juventude influenciam os comportamentos na vida adulta. No presente trabalho, aferimos a classe sócio-ocupacional na juventude relativa ao primeiro emprego, e a média etária de início das atividades laborais foi de 17 anos ( $\mathrm{DP}=4,76$ ). Ou seja, a média etária coincide com a adolescência para a maioria dos participantes deste estudo. Após ajustes mútuos, observamos que a exposição à desvantagem socioeconômica na juventude foi associada de forma independente apenas à maior chance de ser extabagista em homens e mulheres e de tabagismo atual, entre mulheres. Os demais fatores de risco não foram associados à posição socioeconômica na juventude. A posição socioeconômica na adolescência já foi indiretamente relacionada à condição de tabagista na vida adulta 44, e o ex-tabagista também foi tabagista um dia.

Nossos resultados apontaram que os indivíduos que descenderam das classes alta/média para a baixa apresentaram maior chance de comportamentos não saudáveis do que aqueles que sempre pertenceram à classe alta, de forma ainda mais consistente no sexo masculino. Apenas a trajetória ascendente para a classe sócio-ocupacional alta parece não alterar a adoção de comportamentos saudáveis na vida adulta, já que a chance desses comportamentos não diferiu estatisticamente dos que sempre estiveram na posição alta. Esses achados sugerem que os indivíduos, ao migrarem para um novo grupo social, tendem a mimetizar hábitos de seu novo grupo, considerando que os grupos em posição sócio -ocupacional inferior têm maiores prevalências desses comportamentos como indicam nossa análise por períodos de vida e achados de outros autores 15,17. Verificamos que homens e mulheres que permaneceram na classe baixa inferior da juventude até a vida adulta têm, em geral, as maiores chances de apresentarem comportamentos danosos para a saúde. Resultados semelhantes foram observados entre mulheres do Reino Unido 15 e em uma revisão sistemática baseada em seis estudos conduzidos na Europa 17. De certa forma, esse resultado sugere que as chances de comportamentos de risco aumentam à proporção que a duração da exposição às adversidades sociais persistem ao longo da vida 45, o que poderia indicar um efeito cumulativo da baixa posição socioeconômica. Diferentemente da ascensão para a classe alta, a ascensão da classe sócio-ocupacional baixa para a média na juventude não resultou em menor chance de comportamentos não saudáveis na vida adulta, possivelmente porque a inserção na classe média foi associada com maior chance de comportamentos de risco na vida adulta na análise que apresentamos por períodos de vida. 
A abordagem do curso de vida oferece a possibilidade de investigar se desvantagens vivenciadas em períodos específicos estão associadas à maior frequência de comportamentos relacionados à saúde na vida adulta. Os comportamentos relacionados à saúde são fortemente influenciados pelo contexto atual, sendo passíveis de mudanças. A grande produção de evidências científicas tem contribuído para adoção de políticas públicas de promoção de modos de vida saudável com impactos sobre a saúde, como a queda na prevalência de tabagismo no Brasil 42. Ressalta-se que apesar disso, as desigualdades sociais no tabagismo, iniciadas e ampliadas a partir da década de 1980 42, não apenas persistem, como têm aumentado 28. Talvez, por essas características modificáveis dos comportamentos, nossos resultados mostrem associações mais consistentes entre a posição socioeconômica atual, de certa forma reforçadas pela análise de trajetória sócio-ocupacional.

Limitações relativas aos resultados do presente trabalho devem ser consideradas. Os comportamentos de saúde foram mensurados em um único momento da vida, mudanças podem ter ocorrido, por motivos de saúde ou por influência de ações de prevenção e promoção da saúde, podendo ambas serem distintas segundo a posição socioeconômica. Os participantes do ELSA-Brasil são servidores públicos, com empregos estáveis, renda e escolaridade média superiores à média nacional. Dessa forma, é possível que indivíduos expostos à extrema adversidade social ao longo da vida não estejam incluídos no presente estudo e que as associações observadas estejam subestimadas. A forma de coleta de dados, autorreferida, pode ser influenciada pelo desejo de transmitir uma imagem social e culturalmente aceitável (viés de desejabilidade social), além disto, a mensuração da atividade física no lazer por meio de entrevista tem menor acurácia do que de forma objetiva 46. A posição socioeconômica na infância e na juventude, mensurada pela escolaridade materna e classe sócio-ocupacional da primeira ocupação do participante foi obtida retrospectivamente, o que pode trazer alguma imprecisão. Se essa imprecisão for não diferencial, como supomos, estaremos subestimando nossas associações. Considere-se também que a escolaridade materna pode ter aumentado ao longo do tempo, mas isto levaria a subestimar a exposição à baixa escolaridade manteria na infância, e, portanto, enviesar as associações observadas em direção à nulidade. Poucos trabalhos examinaram a associação da posição socioeconômica na infância e comportamentos de risco relacionados à saúde, e as diferentes medidas de posição socioeconômica utilizadas nos estudos podem restringir as comparações e consistência dos resultados. Foi realizada análise de sensibilidade restringindo as análises aos participantes com até 64 anos de idade, uma vez que idosos tendem a adotar comportamentos mais saudáveis, no entanto, as alterações observadas nos odds ratio (OR) foram negligenciáveis e as direções às associações não foram alteradas. Por isso, mantivemos os resultados para todas as faixas etárias.

Nossos resultados acrescentam evidências que comportamentos de risco expressam as circunstâncias sociais em que os indivíduos vivem e cresce. As condições de vida moldam condutas e criam oportunidades mais ou menos saudáveis ao longo da vida. Corroboram a maior prevalência de comportamentos de risco nos homens do que em mulheres. Mostram ainda que desvantagens socioeconômicas na vida adulta parecem exercer maior influência sobre os comportamentos relacionados à saúde do que as desvantagens socioeconômicas na infância e na juventude. As circunstâncias sociais na infância foram mais relevantes para as mulheres, mas de forma contraditória, visto que estiveram associadas à menor chance de tabagismo e maior chance de inatividade física no lazer. Melhorar as desigualdades socioeconômicas poderá impactar nos comportamentos relacionados à saúde e, consequentemente, a morbimortalidade por doenças crônicas. 


\section{Colaboradores}

J. C. Faleiro, L. Giatti, S. M. Barreto e L. V. Camelo foram responsáveis pela redação, desenho, análise e interpretação dos resultados e revisão crítica. $\mathrm{R}$. H. Griep, J. M. N. Guimarães, M. J. M. Fonseca, D, Chor e M. C. A. Chagas contribuiram com a interpretação dos resultados e revisão crítica.

\section{Referências}

1. Glorioso V, Pisati M. Socioeconomic inequality in health-related behaviors: a lifestyle approach. Qual Quant 2014; 48:2859-79.

2. Wardle J, Steptoe A. Socioeconomic differences in attitudes and beliefs about healthy lifestyles. J Epidemiol Community Health 2003; 57:440-3.

3. Nevill A, Donnelly P, Shibli S, Foster C, Murphy M. Modifiable behaviors help to explain the inequalities in perceived health associated with deprivation and social class: evidence from a national sample. J Phys Act Health 2014; 11:339-47.

4. Stringhini S, Sabia S, Shipley M, Brunner E, Nabi H, Kivimaki M, et al. Association of socioeconomic position with health behaviors and mortality. JAMA 2010; 303:1159-66.

\section{Agradecimentos}

Os autores agradecem à equipe e aos participantes do ELSA-Brasil por suas importantes contribuições. O estudo foi apoiado pelos Ministérios da Saúde (Departamento de Ciência e Tecnologia) e da Ciência e Tecnologia (FINEP/CNPq) do Brasil. S. M. B., L. G., D. C. e R. H. G. são bolsistas de produtividade em pesquisa do Conselho Nacional de Desenvolvimento Científico e Tecnológico (CNPq: 300159/99-4, 312371/13-6, 303371/2014-5, 303251/2013-1, respectivamente). L. V. C. é bolsista de pós-doutorado do Programa Nacional de Pósdoutorado da Coordenação de Aperfeiçoamento de Pessoal de Nível Superior (PNPD-Capes). A linha de base do ELSA-Brasil foi financiada pelo Ministério da Saúde.
5. United Nations Development Programme. Discussion paper: addressing the social determinants of noncommunicable diseases. New York: United Nations Development Programme; 2013.

6. Galobardes B, Shaw M, Lawlor DA, Lynch JW, Smith GD. Indicators of socioeconomic position (part 1). J Epidemiol Community Health 2006; 60:7-12.

7. Braveman PA, Egerter SA, Mockenhaupt RE. Broadening the focus: the need to address the social determinants of health. Am J Prev Med 2011; 40(1 Suppl 1):S4-18. 
8. Cockerham WC. Health lifestyle theory and the convergence of agency and structure. J Health Soc Behav 2005; 46:51-67.

9. Lynch JW, Kaplan GA, Salonen JT. Why do poor people behave poorly? Variation in adult health behaviours and psychosocial characteristics by stages of the socioeconomic lifecourse. Soc Sci Med 1997; 44:809-19.

10. Dorner APDT, Hoffmann K, Stein KV, Niederkrotenthaler T. Socio-economic determinants of health behaviours across age groups: results of a cross-sectional survey. Wien Klin Wochenschr 2013; 125:261-9.

11. Lv J, Liu Q, Ren Y, Gong T, Wang S, Li L, et al. Socio-demographic association of multiple modifiable lifestyle risk factors and their clustering in a representative urban population of adults: a cross-sectional study in Hangzhou, China. Int J Behav Nutr Phys Act 2011; 8:40.

12. Tehranifar P, Liao Y, Ferris JS, Terry MB. Life course socioeconomic conditions, passive tobacco exposures and cigarette smoking in a multiethnic birth cohort of US women. Cancer Causes Control 2009; 20:867-76.

13. Atkins JL, Ramsay SE, Whincup PH, Morris RW, Lennon LT, Wannamethee SG. Diet quality in older age: the influence of childhood and adult socio-economic circumstances. Br J Nutr 2015; 113:1441-52.

14. Hare-Bruun H, Togo P, Andersen LB, Heitmann BL. Adult food intake patterns are related to adult and childhood socioeconomic status. J Nutr 2011; 141:928-34.

15. Watt HC, Carson C, Lawlor DA, Patel R, Ebrahim S. Influence of life course socioeconomic position on older women's health behaviors: findings from the British Women's Heart and Health Study. Am J Public Health 2009; 99: 320-7.

16. Power C, Graham H, Due P, Hallqvist J, Joung I, Kuh D, et al. The contribution of childhood and adult socioeconomic position to adult obesity and smoking behaviour: an international comparison. Int J Epidemiol 2005; 34:335-44.

17. Motta JVDS, Lima NP, Olinto MTA, Gigante DP. Social mobility and smoking: a systematic review. Ciênc Saúde Coletiva 2015; 20:1515-20.

18. Karvonen S, Rimpelä AH, Rimpelä MK. Social mobility and health related behaviours in young people. J Epidemiol Community Health 1999; 53:211-7.

19. Chapman BP, Fiscella K, Duberstein P, Kawachi I, Coletta M. Can the influence of childhood SES on men and women's adult body mass be explained by adult SES or personality? Findings from a national sample. Health Psychol 2009; 28:419-27.

20. Osler M, Godtfredsen NS, Prescott E. Childhood social circumstances and health behaviour in midlife: the Metropolit 1953 Danish male birth cohort. Int J Epidemiol 2008; 37:1367-74.

21. Schmidt MI, Duncan BB, Mill JG, Lotufo PA, Chor D, Barreto SM, et al. Cohort profile: longitudinal study of adult health (ELSA-Brasil). Int J Epidemiol 2015; 44:68-75.
22. Campbell DJ, Ronksley PE, Manns BJ, Tonelli M, Sanmartin C, Weaver RG, et al. The association of income with health behavior change and disease monitoring among patients with chronic disease. PLoS One 2014; 9:e94007.

23. International Physical Activity Questionnaire. Guidelines for data processing and analysis of the International Physical Activity Questionnaire (IPAQ): short and long forms. IPAQ Group; 2005. http://www.ipaq.ki.se (acessado em Ago/2008).

24. World Health Organization. Global recommendations on physical activity for health. Geneva: World Health Organization; 2010.

25. Martins TG, Assis MAAD, Nahas MV, Gauche H, Moura EC. Leisure-time physical inactivity in adults and factors associated. Rev Saúde Pública 2009; 43:814-24.

26. Instituto Brasileiro de Geografia e Estatística. Pesquisa Nacional de Saúde: percepção do estado de saúde, estilos de vida e doenças crônicas: 2013. Rio de Janeiro: Instituto Brasileiro de Geografia e Estatística; 2014.

27. De Irala-Estevez J, Groth M, Johansson L, Oltersdorf U, Prattala R, Martínez-González MA. A systematic review of socio-economic differences in food habits in Europe: consumption of fruit and vegetables. Eur J Clin Nutr 2000; 54:706-14.

28. Barros AJ, Cascaes AM, Wehrmeister FC, Martínez-Mesa J, Menezes AMB. Tabagismo no Brasil: desigualdades regionais e prevalência segundo características ocupacionais. Ciênc Saúde Coletiva 2011; 16:3707-16.

29. Iglesias R, Jha P, Pinto M, Silva VLDC, Godinho J. Documento de discussão saúde, nutrição e população (HNP). Controle do tabagismo no Brasil: resumo executivo. Epidemiol Serv Saúde 2008; 17:301-4.

30. Shaw BA, McGeever K, Vasquez E, Agahi N, Fors S. Socioeconomic inequalities in health after age 50: are health risk behaviors to blame? Soc Sci Med 2014; 101:52-60.

31. Camelo LV, Giatti L, Barreto SM. Subjective social status, self-rated health and tobacco smoking: Brazilian Longitudinal Study of Adult Health (ELSA-Brasil). J Health Psychol 2014; 19:1388-99.

32. Pessoa MC, Mendes LL, Gomes CS, Martins PA, Velasquez-Melendez G. Food environment and fruit and vegetable intake in a urban population: a multilevel analysis. BMC Public Health 2015; 15:1012.

33 Dibsdall LA, Lambert N, Bobbin RF, Frewer LJ. Low-income consumers' attitudes and behaviour towards access, availability and motivation to eat fruit and vegetables. Public Health Nutr 2003; 6:159-68.

34. Coelho AB, Aguiar DRDD, Fernandes EA. Padrão de consumo de alimentos no Brasil. Revista de Economia e Sociologia Rural 2009; 47:335-62. 
35. Humpel N, Owen N, Leslie E. Environmental factors associated with adults' participation in physical activity: a review. Am J Prev Med 2002; 22:188-99.

36. Roman CG, Chalfin A. Fear of walking outdoors: a multilevel ecologic analysis of crime and disorder. Am J Prev Med 2008; 34:306-12.

37. Dias-da-Costa JS, Hallal PC, Wells JCK, Daltoé T, Fuchs SC, Menezes AMB, et al. Epidemiology of leisure-time physical activity: a population-based study in southern Brazil. Cad Saúde Pública 2005; 21:275-82.

38. Siahpush M, Jones PR, Singh GK, Timsina LR, Martin J. The association of tobacco marketing with median income and racial/ethnic characteristics of neighbourhoods in Omaha, Nebraska. Tob Control 2010; 19:256-8.

39. Link BG, Phelan J. Social conditions as fundamental causes of disease. J Health Soc Behav 1995; Spec No:80-94.

40. Mckenzie SK, Carter KN, Blakely T, Ivory V. Effects of childhood socioeconomic position on subjective health and health behaviours in adulthood: how much is mediated by adult socioeconomic position? BMC Public Health 2011; 11:269.

41. Lawlor DA, Batty GD, Morton SM, Clark H, Macintyre S, Leon DA. Childhood socioeconomic position, educational attainment, and adult cardiovascular risk factors: the Aberdeen children of the 1950s cohort study. Am J Public Health 2005; 95:1245.
42. Monteiro CA, Cavalcante TM, Moura EC, Claro RM, Szwarcwald CL. Population-based evidence of a strong decline in the prevalence of smokers in Brazil (1989-2003). Bull World Health Organ 2007; 85:527-34.

43. Barreto SM, Figueiredo RC, Giatti L. Socioeconomic inequalities in youth smoking in Brazil. BMJ Open 2013; 3:e003538.

44. Novak M, Hammarstrom A, Ahlgren C. Inequalities in smoking: influence of social chain of risks from adolescence to young adulthood: a prospective population-based cohort study. Int J Behav Med 2007; 14:181-7.

45. Lynch J, Smith GD. A life course approach to chronic disease epidemiology. Annu Rev Public Health 2005; 26:1-35.

46. Hagstromer M, Ainsworth BE, Oja P, Sjostrom M. Comparison of a subjective and an objective measure of physical activity in a population sample. J Phys Act Health 2010; 7:541-50. 


\section{Abstract}

Our objective was to investigate the association between lifetime socioeconomic status and intragenerational social mobility and low consumption of fruits and vegetables, leisure-time physical inactivity, and smoking among 13,216 men and women participating in the baseline of the ELSABrazil study (2008-2010). Socioeconomic status in childhood, adolescence, and adulthood was measured by maternal schooling, socio-occupational class of the first occupation, and socio-occupational class of the current occupation, respectively. Social disadvantages in adulthood were consistently associated with higher prevalence of the three behaviors analyzed in men and women. However, socioeconomic status in youth and childhood was less consistently associated with the behaviors. For example, while low maternal schooling reduced the odds of past smoking (women) and current smoking (men and women), it was associated with higher odds of leisure-time physical inactivity in women. Meanwhile, low socioeconomic status in youth increased the odds of past smoking (men and women) and current smoking (women). Analysis of social trajectories lent additional support to the relevance of disadvantages in adulthood for risk behaviors, since only individuals that rose to the high socio-occupational class did not show higher odds of these behaviors when compared to participants that had always belonged to the high sociooccupational class. Our findings indicate that socioeconomic disadvantages in adulthood appear to be more relevant for risk behaviors than disadvantages in childhood and adolescence.

Social Class; Social Mobility; Health Behavior

\section{Resumen}

Nuestro objetivo fue investigar la asociación de la posición socioeconómica en el curso de vida y de la movilidad social intrageneracional, con el bajo consumo de frutas y hortalizas, inactividad física en el tiempo libre y tabaquismo entre 13.216 hombres y mujeres, participantes de la línea de base del ELSA-Brasil (2008-2010). La posición socioeconómica durante la infancia, juventud y vida adulta se midió por la escolaridad materna, clase socio-ocupacional de la primera ocupación y clase socio-ocupacional de la ocupación actual, respectivamente. Las desventajas sociales en la vida adulta fueron consistentemente asociadas a una mayor prevalencia de los tres comportamientos analizados en hombres y mujeres. Entretanto, la posición socioeconómica en la juventud e infancia se asoció a los comportamientos de forma menos consistentes. Por ejemplo, en cuanto a la baja escolaridad materna redujo la oportunidad de tabaquismo pasado (mujeres) y actual (hombres y mujeres), ésta se asoció a una mayor oportunidad de inactividad física en el tiempo libre entre las mujeres. Ya la exposición a la baja posición socioeconómica en la juventud aumentó las oportunidades de tabaquismo pasado (hombres y mujeres) y actual (mujeres). El análisis de las trayectorias sociales dio apoyo adicional a la mayor importancia de las desventajas en la vida adulta para comportamientos de riesgo, ya que sólo individuos que ascendieron a la clase socio-ocupacional alta no presentaron una mayor oportunidad de estos comportamientos, cuando se comparan con los participantes que siempre pertenecieron a la clase socio-ocupacional alta. Nuestros resultados apuntan que las desventajas socioeconómicas en la vida adulta parecen ser más relevantes para comportamientos de riesgo que las desventajas en la infancia y adolescencia.

Clase Social; Movilidad Social;

Conductas Saludables
Recebido em 03/Fev/2016

Versão final reapresentada em 05/Mai/2016

Aprovado em 08/Jul/2016 